\title{
Electron Microscopy Study of Interfaces of Proton Conducting Fuel Cell Anodes
}

\author{
D.M. Kepaptsoglou*, Q.M. Ramasse**, M.F. Sunding*, S.Diplas***, T. Norby****, A.E. Gunnæs* \\ * Department of Physics, University of Oslo, NO-0316 Oslo, Norway \\ ** National Centre for Electron Microscopy, Lawrence Berkeley National Laboratory, 1 Cyclotron \\ Road, Berkeley, California 94708 \\ *** SINTEF Materials and Chemistry, Forskningsveien 1, Oslo, Norway \\ **** Department of Chemistry and Centre for Materials Science and Nanotechnology (SMN), \\ University of Oslo, P.O. Box 1033 Blindern, NO-0315 Oslo, Norway
}

In the race for clean and efficient production of energy, solid state fuel cells emerge as cutting edge technology with very promising results. High-temperature proton conductors are oxides with oxygen deficiency in the form of dilute oxygen vacancies, where protons dissolve as hydroxide defects in the oxide at the expense of the vacancies. Acceptor doped proton conducting oxides, such as $\mathrm{Ca}$-doped orthoniobates have shown remarkable conduction properties and chemical stability and thus show great potential in their employment as components for solid state fuel cells [1,2]. To a large extent the functionality and overall efficiency of fuel cells is dependent on the nature of oxide/oxide and metal/oxide interfaces in its electrodes, since the current is often limited by the conductivity of the grain boundaries and by electrode kinetics. In particular, the mobility of charge carriers across or along interfaces can be affected by the presence of electrical charging and oxygen vacancies as a result of doping, local structural changes at the interface or the formation of interfacial precipitation layers [3].

The TEAM 0.5 instrument, a modified double-corrected FEI Titan ${ }^{3}$ microscope [4], was used to study the structural and chemical characteristics of the interfaces between $\mathrm{Ni}$ and $\mathrm{LaNbO}_{4}$ grains in $\mathrm{Ni}-\mathrm{LaNbO}_{4}$ cermets, a candidate material for fuel cell anodes [5]. High resolution high angle annular dark field images (HAADF) of the grain boundaries show that the $\mathrm{LaNbO}_{4}$ grains are not perfectly facetted and that the boundaries with the Ni grain are quite irregular due to the presence of a large density of twinned domains within the $\mathrm{LaNbO}_{4}$. The presence of such kinks and structural defects along the interface may affect the concentration of oxygen vacancies at the core of the grain boundary; oxygen deficiency at the grain boundary results into a positive charge barrier and a negative space charge layer with a proton deficiency, both decreasing the proton conductivity of the grain boundary. It should also be noted that no texturing or coincidental site lattice relationship between the $\mathrm{Ni}$ and $\mathrm{LaNbO}_{4}$ was revealed. Electron energy loss spectroscopy (EELS) was used to investigate the precipitation of dopants or formation of secondary phases at the $\mathrm{Ni} / \mathrm{LaNbO}_{4}$ boundaries. Spatially resolved linescans across the boundaries reveal possible diffusion or intermixing (FIG 2) as well as changes in the local chemical states of $\mathrm{Ni}$ and La at the boundary, likely due to the structural defects at the grain boundaries.

[1] R. Haugsrud, T. Norby, Nat. Mater. 5 (2006) 193

[2] H. Fjeld et al, Solid State Ionics, doi:10.1016/j.ssi.2009.04.019

[3] X. Guo, R. Waser, Prog. Mater. Sci. 51 (2006) 151

[4] C. Kisielowski et al, Microsc. Microanal. 462 (2008) 14454 
[5] This work was performed at NCEM, which is supported by the Office of Science, Office of Basic Energy Sciences of the U.S. Department of Energy under Contract No. DE-AC0205CH11231.
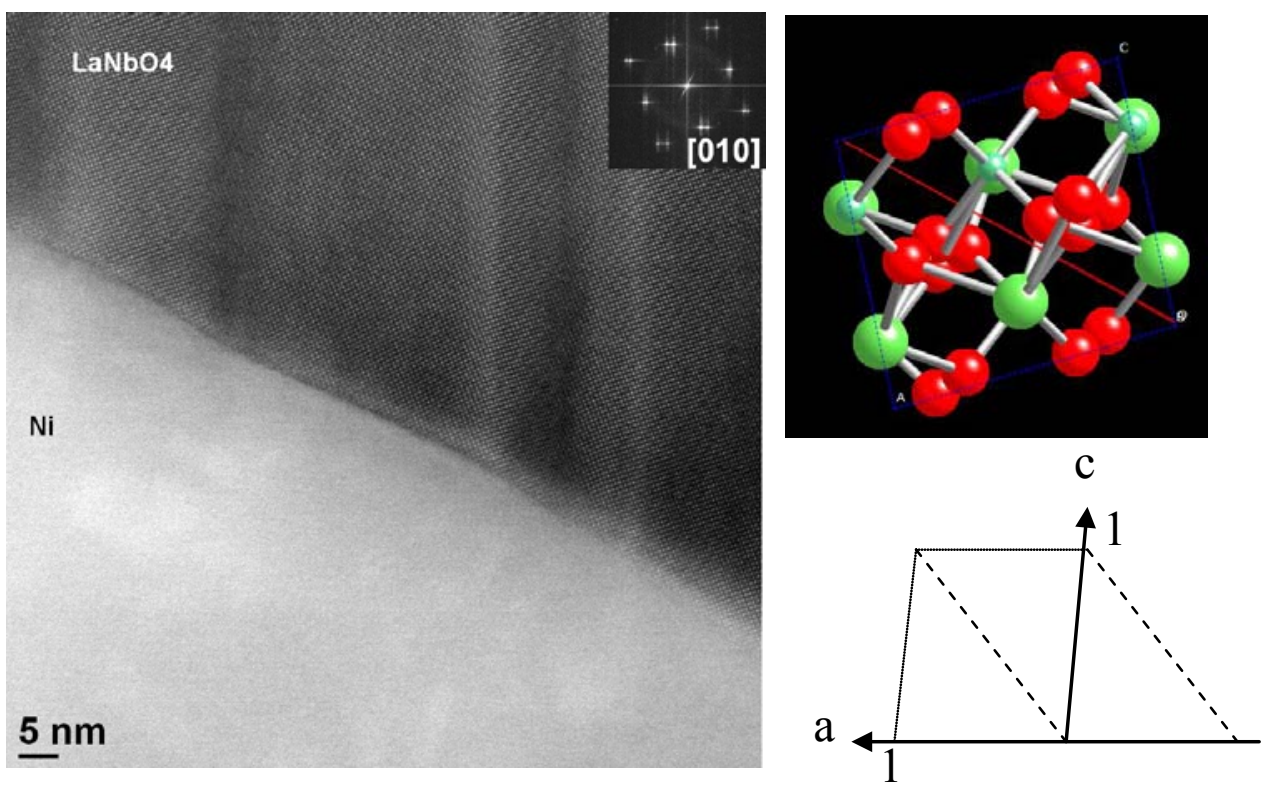

FIG 1. HAADF image of a Ni-LaNbO 4 interface; showing bending of the (-101) $\mathrm{LaNbO}_{4}$ due to the presence of twinned domains.
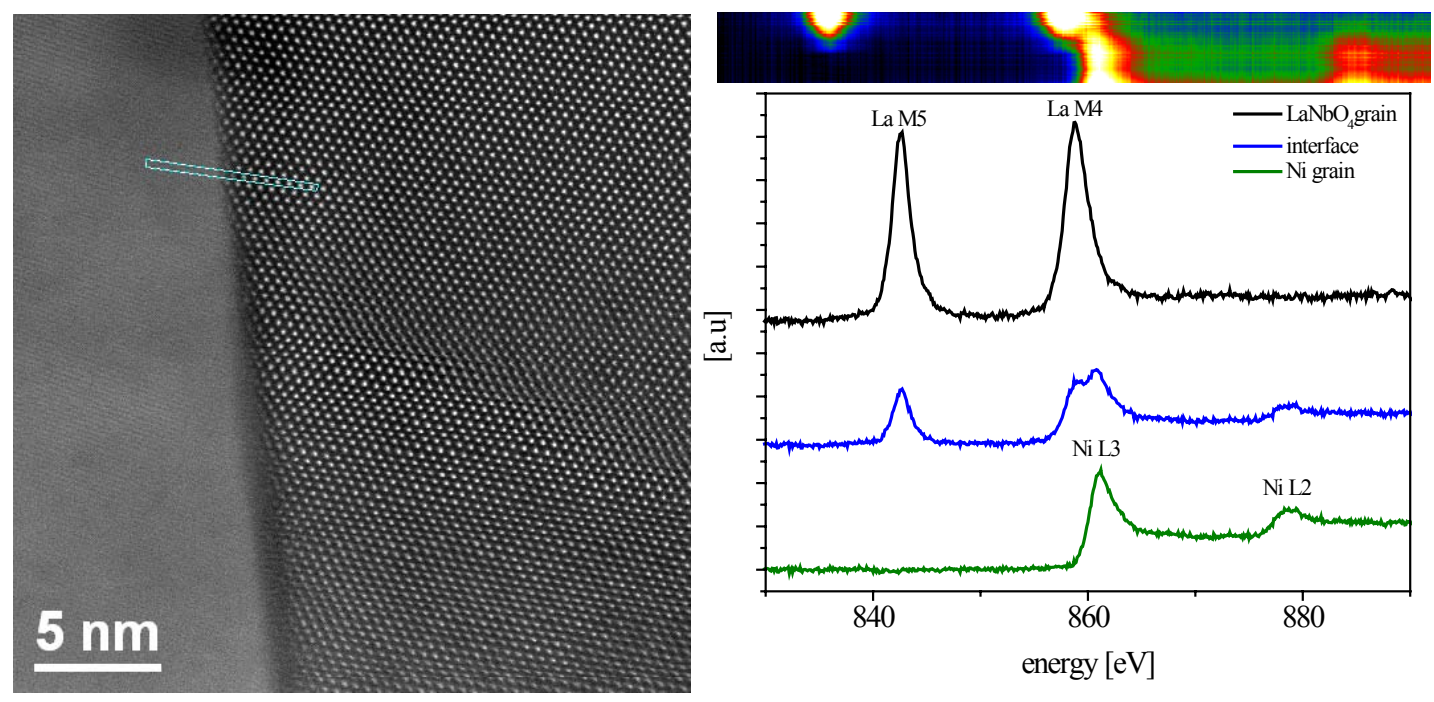

FIG 2. HAADF image of a Ni-LaNbO 4 interface, showing the length of the EELS linescan (left) raw EELS spectrum image (top right) and selected La M5,4 and Ni L3,2 EELS spectra. 